\title{
Favourable pharmacokinetics of intradermal adalimumab over subcutaneous administration: results of a randomized controlled trial
}

Justin Jacobse $^{1}$, Wouter ten Voorde ${ }^{1}$, Anushka Tandon ${ }^{1}$, Stefan Romeijn ${ }^{2}$, Hendrika Grievink $^{1}$, Koen van der Maaden ${ }^{2}$, Michiel van Esdonk ${ }^{3}$, Dirk Jan Moes ${ }^{4}$, Floris Loeff ${ }^{5}$, Karien Bloem ${ }^{5}$, Annick de Vries ${ }^{5}$, Theo Rispens ${ }^{5}$, Gertjan Wolbink ${ }^{5}$, Marieke de Kam ${ }^{1}$, Dimitrios Ziagkos $^{1}$, Matthijs Moerland ${ }^{1}$, Wim Jiskoot ${ }^{2}$, Joke Bouwstra ${ }^{2}$, Jacobus Burggraaf $^{1}$, Robert Rissmann ${ }^{1}$, Lenneke Schrier ${ }^{6}$, and Rebecca Ten Cate ${ }^{6}$

${ }^{1}$ Centre for Human Drug Research

${ }^{2}$ Leiden Academic Centre for Drug Research

${ }^{3}$ Leiden University

${ }^{4}$ Leiden University Medical Center

${ }^{5}$ Sanquin Diagnostic Services

${ }^{6}$ Leiden University Medical Center Willem Alexander Childrens Hospital

September 28, 2020

\begin{abstract}
Aim To evaluate feasibility of intradermal (i.d.) adalimumab administration using hollow microneedles, and to compare a single i.d. dose of adalimumab using a hollow microneedle with a single subcutaneous (s.c.) dose using a conventional needle. Methods In this single-centre double-blind, placebo-controlled, double-dummy clinical trial in 24 healthy adults we compared $40 \mathrm{mg}$ adalimumab $(0.4 \mathrm{~mL})$ administered i.d. using a hollow microneedle with a s.c. dose using a conventional needle. Primary parameters were pain, acceptability, and local tolerability; secondary parameters safety, pharmacokinetics and immunogenicity. We explored usability of optical coherence tomography (OCT), clinical photography, thermal imaging, and laser speckle contrast imaging (LSCI) to evaluate skin reaction after i.d. injections. In vitro protein analysis was performed to assess compatibility of adalimumab with the hollow microneedle device. Results While feasible and safe, injection pain of i.d. adalimumab was higher compared to s.c. adalimumab (35.4 vs. 7.9 on a 101-point VAS scale). Initial absorption rate and bioavailability were higher after i.d. adalimumab $(\operatorname{Tmax}=95 \mathrm{~h}(47-120) ; \mathrm{F}=129 \%(6.46 \%)$ ) compared to s.c. adalimumab $(\operatorname{Tmax}=120 \mathrm{~h}(96-221))$. In $50 \%$ and $83 \%$ of the subjects anti-adalimumab antibodies were detected after i.d. and s.c. adalimumab, respectively. We observed statistically significantly more erythema and skin perfusion after i.d. adalimumab, compared to s.c. adalimumab and placebo injections $(\mathrm{p}<0.0001)$. Cytokine secretion after whole blood LPS challenge was comparable between administration routes. Conclusion Intradermal of adalimumab using hollowing microneedles was perceived as more painful, and less accepted than s.c. administration, however, yields a higher bioavailability with similar safety and pharmacodynamic effects.
\end{abstract}

\section{Introduction}

Biopharmaceuticals, such as monoclonal antibodies (mAbs), are used in the treatment of a large number of chronic and life-threatening diseases (1). Degradation and ineffective absorption of mAbs in the gastrointestinal tract, due to molecular size and conditions such as low $\mathrm{pH}$ and digestive enzymes, necessitates 
their parenteral administration. However, in clinical practice, treatments administered using s.c. injection of $\mathrm{mAbs}$ have been perceived as unpleasant and painful, especially during long term use in both adults and children (2). Thus, s.c. administration may jeopardize treatment adherence and a less invasive and less painful method to administer mAbs is warranted.

Intradermal administration of biopharmaceuticals through hollow microneedles is advocated as substitute for s.c. injection, due to less pain associated with injection of drugs using microneedles (3), and i.d. administered biopharmaceuticals may show more favourable pharmacokinetics as compared to s.c. administration (4-7). Multiple types of microneedles exist, such as hollow and solid microneedles, and microneedles have different properties in comparison with conventional needles. For instance, the injection of pharmaceutical compounds using hollow microneedles is more superficial, i.e., into the skin (i.d.) rather than beneath the skin (s.c.). Additionally, the diameter of hollow microneedles is smaller than that of conventional hypodermic needles for s.c. injection. An unbiased and systematic approach is warranted to acquire reliable data on pain perception and patient preferences, as these are subjective concepts (8). Therefore, it is relevant to compare pain, acceptability and local tolerability, as well as pharmacokinetics (PK) and pharmacodynamics (PD) between mAbs administered i.d. using a hollow microneedle with S.C. injection using a conventional hypodermic needle. Moreover, when using a new drug-device combination, chemistry, manufacturing and control (CMC) aspects need consideration.

The commercially available microneedles used in the clinical trial reported in this paper have been used in various clinical studies (9). Each device consists of three hollow microneedles with a length of $600 \mu \mathrm{m}$; this device is hereafter referred to as hollow microneedle. Although microneedle vaccine administration has been widely investigated, there are no systematic reports on $\mathrm{mAb}$ administration using microneedles in humans.

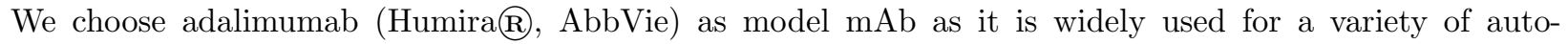
immune/auto-inflammatory diseases including juvenile idiopathic arthritis. Adalimumab acts by binding to the pro-inflammatory cytokine $\mathrm{TNF} \alpha$, hereby preventing its interaction with the TNF $\alpha$ receptor (10).

To evaluate feasibility of i.d. adalimumab administration using hollow microneedles, we performed a doubleblind, double-dummy, randomized controlled clinical trial in healthy adults, comparing a single i.d. dose of adalimumab using a hollow microneedle with a single s.c. dose using a conventional needle. Our primary aim was to systematically investigate pain, acceptability, and local tolerability after i.d. adalimumab administration and to compare this with S.C. administration. Our secondary aim was to evaluate safety, PK, $\mathrm{PD}$, and immunogenicity of i.d. adalimumab administration and to compare this with s.c. administration. Moreover, we explored the usability of optical coherence tomography (OCT), clinical photography, thermal imaging, and laser speckle contrast imaging (LSCI) in the evaluation of i.d. injections. Lastly, prior to the clinical trial we performed an elaborate in vitro protein analysis to confirm that examine whether ejection of adalimumab through a hollow microneedle bore increases particle formation or protein aggregation compared to ejection through a conventional needle. One could envision that during ejection of a protein out of a narrow microneedle, the structure of the protein might be affected. Factors contributing to the immunogenicity of mAbs include protein structure and physical degradation, such as aggregation (11). The formation of anti-adalimumab antibodies may result in reduced treatment efficacy due to increased drug clearance (CL) $(12,13)$.

Altogether, in this paper we provide a systematic and comprehensive approach to answer the question whether hollow microneedles can be used safely and effectively to administer a model mAb.

\section{Methods}

\section{Study design}

This was a single-center double-blind, placebo-controlled, double-dummy clinical trial with four interventions: i.d. adalimumab (40 mg Humira, AbbVie, Maidenhead, UK), i.d. saline (0.9\%), s.c. adalimumab, and s.c. saline. i.d. injections were given using a hollow microneedle (MicronJet600 from Nanopass Technologies Ltd., Ness Ziona, Israel), s.c. injections using a regular needle (Microlance 3 from Becton, Dickenson, and Company (BD), Franklin Lakes, United States of America); both devices were connected to a syringe (1 
$\mathrm{mL}$ Luer-Lok $\left.{ }^{\mathrm{TM}}, \mathrm{BD}\right)$. The length of the three needles of a MicronJet600 device is $600 \mu \mathrm{m}$. Injections were given according to standard operating procedures and the manufacturer's instruction. All subjects received one placebo injection and one adalimumab injection of $40 \mathrm{mg}$ in $0.4 \mathrm{ml}$ in the right and left upper lateral thigh by the same physician. Given the nature of the study, the physician administering the injection could not be blinded to the method of administration but was blinded to treatment, i.e., adalimumab or placebo. Therefore, this physician was not involved in the assessment of any of the pre-defined outcomes (evaluator-blinded). The subjects were in a prone position during and in between injections to ensure blinding (subject-blinded). Injections were spaced five minutes apart. Prior to administration, the sites of injection were annotated using a surgical marker (Purple Surgical, Shenley, England). Subjects were instructed to maintain the marking while at home, and to prevent excessive sun exposure to the injection site to limit possible interference with the exploratory measurements.

\section{Participants}

Twenty-four healthy immunocompetent male and female subjects aged between 18-45 years with Fitzpatrick skin type I-II (Caucasian) and not smoking more than 10 cigarettes per day were included in the study. The ratio male:female was 1:1. Subject health status was verified during a medical screening consisting of a medical history, physical examination, vital signs, 12-lead electrocardiogram, laboratory analysis of blood and urine and a Mantoux and/or interferon gamma release assay. Subjects with a history of tuberculosis were excluded. Routine safety assessments were performed as described earlier (14). Total observation time was 70 days.

\section{Sample size and randomization}

Due to the explorative character of this trial, empirical, early clinical phase cohort sizes were used to answer the objectives of the trial. No formal power calculation was performed. A total of 24 subjects were studied (allocation s.c.: i.d. = 1:1); i.e., 12 subjects received i.d. adalimumab and s.c. placebo and 12 subjects received s.c. adalimumab and i.d. placebo. The sequence of injection, i.e., s.c. followed by i.d. injection or i.d. injection followed by s.c. injection, was counterbalanced. Randomization was done in 6 blocks of 4 , each 4 arms containing one of each 4 arms (adalimumab s.c. followed by placebo i.d.; placebo i.d. followed by adalimumab s.c.; placebo s.c. followed by adalimumab i.d.; adalimumab i.d. followed by placebo s.c.). The randomization code was generated using SAS 9.4 by a study-independent statistician; treatment allocation was only revealed after completion of blind data review and locking of the data. After screening and assessment for suitability, subjects were enrolled in the trial by a physician. Interventions were assigned to subjects by a study-independent statistician.

\section{Outcome measures}

A subjective evaluation of spill was performed by visual inspection of the injection site, estimating the volume that was not injected as percentage of the intended injection volume: no spill; minor spill: $15 \%$ spillage; major spill: $15-50 \%$ spillage; critical spill: $>50 \%$ spillage. Microneedles were inspected post-injection for damage using bright field microscopy.

Pain, acceptability, and local tolerability after i.d. and s.c. adalimumab administration

To quantify pain, visual analogue scores (VAS) using both a $10 \mathrm{~cm}$ visual analogue score (VAS) and the Dutch Faces Pain Scales Revised (FPSR) (15) for pain were completed by the volunteers at screening for the Mantoux, or saline if no Mantoux was given, at the time of drug administration, and after drug administration. Pain scores were obtained separately for insertion of the needle (insertion pain) and infusion of the formulation (infusion pain). A standardized injection site examination was performed to evaluate injection sites. Pain was graded as (0) Absent; (1) Present; no limitations in Activity of Daily Living (ADL); (2) Present; Limitations in age-appropriate instrumental ADL or requires repeated non-narcotic pain reliever (3) Present; Limitations in self-care ADL or interferes with sleep or requires repeated narcotic pain reliever. Induration was scored similarly as injection site pain, but with grade (3) instead being Limitations in selfcare ADL or requires systemic treatment. Tenderness was graded as (0) Absent; (1) Mild discomfort with 
pressure; (2) Discomfort with touch; (3) Discomfort elicited by clothing or bed sheets. Pruritus was graded as (0) Absent; (1) Present, but minimally distracting; (2) Present, distracting during routine activities; (3) Interferes with sleep. Erythema, blister, ulceration, necrosis, and ecchymosis were measured if present.

Subject preference for injection was examined using multiple choice questions. Subjects were asked how they experienced the injections, how they would like to receive a potential future injection, and if they feared the injection(s), using the following options: Do you prefer the first injection, the second injection or do you not have a preference? Subjects were additionally asked whether they had fear or no fear. These questions were asked directly after injection, i.e., before subjects were able to see the injection, and one day after the injections.

Safety, PK, and immunogenicity of i.d. and s.c. adalimumab administration

Adverse events were summarized by treatment group, in subsets of all treatment-emergent AEs, and separately for treatment-related AEs. Clinical laboratory and vital sign measurements were summarized by treatment and change from baseline was recorded. Summary statistics included number of subjects, mean, median, minimum, and maximum values (with standard deviation [SD]). Immunogenicity, i.e., anti-adalimumab antibodies, was reported descriptively.

For PK analyses, serum adalimumab concentrations were assessed in blood collected in $4 \mathrm{~mL}$ plain tubes (BD) after coagulation (30-60 minutes) and centrifugation (2000G for 10 minutes at $4^{\circ} \mathrm{C}$ ), from day 1 (predose) till day 71 post-dose. Adalimumab levels were quantified by fully automated ELISA as described (16) . Briefly, TNF was indirectly coated on microtitre plates. Serum was added and incubated. Immobilized adalimumab was subsequently detected using biotinylated rabbit anti-idiotype. The LOD for this assay is $10 \mathrm{ng} / \mathrm{mL}$.

Anti-adalimumab antibodies were measured using a semi-quantitative radioimmunoassay as previously described (16). Briefly, samples were incubated with Sepharose-immobilized protein A (1.0 mg/test; Pharmacia Uppsala, Sweden) on its surface to capture IgG. After washing, radioactive iodine labelled F(ab')2 fragments of adalimumab were added to detect drug-specific antibodies. The LOD for this assay $12 \mathrm{AU} / \mathrm{mL}$.

Ex vivo whole blood challenge was performed to assess the effect of adalimumab on the release of cytokines by circulating immune cells and activation of these cells. Blood $(6 \mathrm{~mL})$ was collected in sodium heparin tubes (Becton Dickinson, NJ, USA) followed by stimulation with $2 \mathrm{ng} / \mathrm{mL}$ lipopolysaccharide (Sigma-Aldrich, Deisenhofen, Germany) and $25 \mu \mathrm{g} / \mathrm{mL}$ aluminium hydroxide (Alhydrogel 2\%, Invivogen, Toulouse, France) for 24 hours at $37^{\circ} \mathrm{C}, 5 \% \mathrm{CO}_{2}$. Culture supernatants were assayed for release of pro-inflammatory cytokines TNF $\alpha$, IL-6, IL-1 $\beta$, IFN $\gamma$ and IL-8 using the Mesoscale Discovery multiplex immunoassay platform.

Usability of optical coherence tomography, clinical photography, thermal imaging, and laser speckle contrast imaging in the evaluation of i.d. injections

Subjects were acclimatized in a temperature-controlled room $\left(21^{\circ} \mathrm{C}\right)$ for 15 minutes with bare legs. The sequence of measurements was (starting with the least invasive to minimize disturbance of the subsequent measurements): (1) thermography; (2) cutaneous micro circulation; (3) 3D photography; (4) multispectral imaging; and (5) skin morphology. Details of skin imaging methods are described below.

Skin micro circulation was quantified by LSCI (PeriCam PSI NR system, Perimed, Sweden). Laser speckle is the interference pattern returning from erythrocytes, resulting in a speckle pattern that differs under changes in blood flow (17). Recordings of 40 seconds were taken from a distance of $15 \mathrm{~cm}$ with a reading frame of 7 by $7 \mathrm{~cm}$. Analysis was performed using the internal software (PimSoft, Perimed, Sweden) and regions of interest were selected based on the most predominant injection site reaction.

Skin temperature was quantified by infrared thermography (FLIR X6540sc camera, FLIR Systems Inc., USA). After calibration for room temperature using a black body, 10 second recordings were taken from a distance of $80 \mathrm{~cm}$. Recordings were averaged for analysis. 
Skin morphology was assessed by OCT (D-OCT VivoSight, Michelson Diagnostics, UK). Thirty second scans were performed with a $6 \mathrm{~mm}$ diameter probe. Three automatically calculated parameters were used to quantify morphology (attenuation compensation, blood flow at depth and skin roughness). Qualitative analysis was performed by two clinical scientists with experience in analyzing D-OCT images.

Erythema and swelling were quantified using a multispectral camera (Antera 3D, Miravex, Ireland), and a 3D stereophotogrammetry camera (3D LifeViz, QuantifiCare, USA). The multispectral camera was placed over the skin creating a closed environment with the lesion in the centre of the frame. Erythema was measured using the CIELab *a value. CIELab is a standardized quantitative method to discriminate colours using an XYZ-axis system. CIELab *a value is represented on the red/green axis and is correlated to skin erythema $(18,19)$. Three-dimensional images were taken from a distance of $20 \mathrm{~cm}$ with use of a guidance laser and analyzed in imaging processing software (DermaPix Software, QuantifiCare, Valbonne, France).

In vitro protein analysis

Adalimumab $100 \mathrm{mg} / \mathrm{mL}$ pre-filled pens or syringes (depending on availability) of the same batch and expiration date were pooled. Storage containers were: (1) syringe only, (2) Verex $2 \mathrm{ml}$ clear glass vial (Phenomenex, Torrance, CA, USA); (3) syringe with a MicronJet600. For condition (1) a capped regular needle was attached during storage to prevent evaporation. Samples were measured immediately (to assess the effect of repackaging), or after storage for four hours at $4^{\circ} \mathrm{C}$ (to assess in-use stability). Directly before analysis the samples were ejected from the syringe into a glass vial and subsequently diluted to $10 \mathrm{mg} / \mathrm{mL}$ or $1 \mathrm{mg} / \mathrm{mL}$ with solvent. The solvent consisted of Milli-Q water with $1.2 \mathrm{~g}$ per $100 \mathrm{~mL}$ mannitol (Sigma, St Louis, MO, USA) and $100 \mathrm{mg} / 100 \mathrm{~mL}$ polysorbate 80 (Sigma, St Louis, MO, USA), and was filtered through an Anotop $10 \mathrm{~mm}, 0.1 \mu \mathrm{m}$ syringe filter (Whatman, Maidstone, UK) before use. For NTA optimization, the solvent was made without polysorbate 80 . Experiments were performed at room temperature, and in a dust free cabinet whenever possible. Changes in protein conformation were determined by second-derivative UV spectroscopy. The formation of adalimumab aggregates and particles was determined by dynamic light scattering (DLS), high-pressure size-exclusion chromatography (HP-SEC), Micro-Flow Imaging (MFI), and nanoparticle tracking analysis (NTA) as described (20) and summarized below.

\section{UV spectroscopy}

Second-derivative UV spectroscopy was used to detect conformational changes. Measurements were performed on an Agilent $8453 \mathrm{UV}-\mathrm{Vis}$ spectrometer (Agilent Technologies, Waldbronn, Germany). Samples were measured in $2 \mathrm{ml}$ half-micro quartz cuvettes (Hellma Benelux, Kruibeke, Belgium) with a path length of $10 \mathrm{~mm}$ in a concentration of $1 \mathrm{mg} / \mathrm{mL}$. Absorbance was measured from 248 to $332 \mathrm{~nm}$ with $1 \mathrm{~nm}$ intervals and an integration time of 15 seconds. Background correction was performed using solvent. Second-derivative spectra were calculated with UV-Visible ChemStation software (Agilent Technologies, Walbronn, Germany) as described earlier (20). The a/b ratio, i.e., the ratio between (a) the vertical distance between the peak minimum at $283 \mathrm{~nm}$ and the maximum at $287 \mathrm{~nm}$ and (b) the vertical distance between the minimum and maximum at $290 \mathrm{~nm}$ and $295 \mathrm{~nm}$ was calculated and used to determine the exposure of tyrosine residues to bulk solvent, which is sensitive to changes in the tertiary structure (21).

\section{Dynamic light scattering}

DLS was used to detect aggregates in the size range from about $1 \mathrm{~nm}$ to $1 \mu \mathrm{m}$. DLS was performed on a Malvern Zetasizer Nano (Malvern, Herrenberg, Germany). $500 \mu \mathrm{L}$ of each sample in a concentration of $10 \mathrm{mg} / \mathrm{mL}$ was analyzed in plastic cuvettes at $25^{*} \mathrm{C}$ using the automatic mode $(\mathrm{n}=3)$. Z-average diameter and polydispersity index were calculated using Dispersion Technology Software version 7.03 (Malvern, Herrenberg, Germany).

\section{High-pressure size exclusion chromatography}

HP-SEC was used to quantify the amount of monomers, dimers, and fragments. Adalimumab samples of $1 \mathrm{mg} / \mathrm{ml}$ were injected in a volume of $50 \mu \mathrm{l}$ onto a SRT SEC-300, $5 \mu \mathrm{m}, 30 \mathrm{~cm} \mathrm{x} 7.8 \mathrm{~mm}$ column (Supelco, Bellefonte, PA, USA). An Agilent 1200 chromatography system (Agilent Technologics, Palo Alto, 
California) combined with an Agilent $1200 \mathrm{UV}$ detector and a multi-angle laser light scattering detector (DAWNR HELEOS, Wyatt Technology Europe GmbH) was used. The flow rate was $0.5 \mathrm{~mL} / \mathrm{min}$. The mobile phase was composed of $50 \mathrm{mM}$ phosphate, $150 \mathrm{mM}$ arginine and $0.025 \% \mathrm{NaN} 3$ at $\mathrm{pH}$ 6.5. To quantify aggregation, UV absorption at $280 \mathrm{~nm}$ was recorded. From the MALLS signal, the root mean square diameter was calculated using the Berry Fit in the Astra software version 5.3.2.22 (Wyatt Technology Europe GmbH, Dernbach, Germany).

\section{Micro-Flow Imaging}

MFI was used to detect particles up to $70 \mu \mathrm{m}$. A MFI5200 (ProteinSimple, Santa Clara, USA), equipped with a silane coated flow cell $(1.41 \times 1.76 \times 0.1 \mathrm{~mm})$ and controlled by the MFI View System Software version 2 was used. Prior to each measurement the system was flushed with purified water. The background was zeroed by using solvent and performing the optimize illumination procedure. Samples of $1 \mathrm{mg} / \mathrm{mL}$ adalimumab were analyzed without a predefined pre-run volume because of the limited amount. Flow rate was $0.17 \mathrm{ml} / \mathrm{min}$ and camera shot rate was 22 flashes per second. Data was analyzed with MFI View Analysis Suite version 1.2. For each product, stuck, edge, and slow-moving particles were removed by the software before analysis. Because no pre-run volume could be used, the data was recorded throughout the entire run but processed only in the time window from 0.7 to $1.7 \mathrm{~min}$ where, based on the trend chart option in the software, the measurement was stable for all samples. The equivalent circular diameter was calculated as described earlier (20).

\section{Nanoparticle tracking analysis}

NTA was used to detect particles between about 50 and $1000 \mathrm{~nm}$. Measurements were performed with a NanoSight LM20, equipped with a sample chamber with a 635-nm laser for illumination of the particles. Samples of $10 \mathrm{mg} / \mathrm{mL}$ adalimumab were injected into the chamber by an automatic pump (Harvard Apparatus, catalog no. 984362, Holliston, USA) using a sterile $1 \mathrm{ml}$ syringe (BD Discardit II, Franklin Lakes, New Jersey). For each sample a 90 second video was captured with the shutter set at $29.9 \mathrm{~ms}$ and the gain at 680. Videos were analyzed using NTA 2.0 Build 127 software. The following settings were used for tracking of the particles: background extract on; brightness 0; gain 1; blur size 3x3; detection threshold 10, viscosity 0.953. All other parameters were set to the automatic adjustment mode.

\section{Statistics}

The population analyzed for pain, tolerability, preference, skin imaging, and pharmacodynamic endpoints included all randomized subjects $(\mathrm{n}=24$ subjects). The population analyzed for PK parameters and PK modelling included injections in which no spillage during treatment administration was reported $(\mathrm{n}=$ 43 injections). Repeated pain injection data (VAS and FPSR) were analyzed with a repeated measures ANOVA with fixed factors treatment, method, time, treatment by method, treatment by time, method by time, treatment by method by time, random factor subject and repeated factor time within subject by treatment by method. The injection pain score of the Mantoux intradermal injection at screening was used as covariate. Single measured pain insertion data (VAS and FPSR) were analyzed with a repeated measures model ANOVA with fixed factors treatment, method, treatment by method, and repeated factor method within subject. The insertion pain score of the Mantoux intradermal injection at screening was used as covariate. Repeated cytokines data were analyzed with a repeated measures ANOVA with fixed factors method, time, method by time, repeated factor time within subject and the baseline as covariate. The contrasts of interest were s.c. vs. i.d. and s.c. vs. i.d. within compound. For imaging analyses a subset of data was used as some variables were zero in some conditions or timepoints. If applicable, the factors of the mixed model were adjusted.

\section{Pharmacokinetic analyses}

Pharmacokinetic parameters derived from serum sample concentrations were calculated using a noncompartmental analysis (NCA). (NCA). The NCA was performed using R version 3.5.3 (22) while the linear trapezoid rule was used for the calculation of AUCs. Analysis of the differences between methods were based 
on least squares means from the ANOVA of the ln-transformed $\mathrm{AUC}_{0-\mathrm{t}} \mathrm{AUC}_{0 \text {-inf }}$, and $\mathrm{C}_{\max }$. In addition, Wilcoxons tests were performed on $\mathrm{T}_{\max }$.

\section{Population PK modelling}

The identification of structural differences in the PK properties of s.c. and i.d. administration, while accounting for covariates such as the presence of anti-adalimumab antibodies, was investigated using a population non-linear mixed effects modelling approach in NONMEM (ICON plc, V7.3). Based on literature information, a one compartment structural model with linear absorption and linear elimination was used during model development (23). For this structural model, the effect of anti-drug antibodies on the CL of adalimumab was tested as a time-varying covariate, increasing the CL of adalimumab at higher titre levels with the following equation: $\mathrm{CL}=\Theta \mathrm{TV}_{\mathrm{CL}} *\left(1+\Theta \mathrm{TV}_{\text {Titre-slope }} * \mathrm{TITRE}\right)$, Where individual TITRE levels proportionally increase the CL of an individual over time.

When a structural misspecification was identified in the absorption phase, modifications to the absorption part of the model were explored, in which transit models, different absorption compartments, and a MTIME function in which the $\mathrm{k}_{\mathrm{a}}$ changes after an estimated time point, were investigated, modelled separately for each administration route.

After identification of the best structural absorption models for each route of administration, log-transformed inter-individual variability (IIV) was included following a forward inclusion procedure $(\mathrm{p}<0.01)$ and covariates (age, weight, body mass index, sex, serum creatinine, and albumin) were explored following a forward-inclusion $(\mathrm{p}<0.01)$ with backward-elimination $(\mathrm{p}[?] 0.001)$ procedure. Continuous covariates were tested following a power relationship centered around the median. Models were evaluated on basis of the objective function value (OFV), the parameter uncertainty (judged by the relative standard error [RSE]), goodness-of-fit figures, individual model predictions versus observations over time, and confidence interval visual predictive checks (ciVPC) based on 500 Monte Carlo simulations. Bootstrapping was not considered of added value as additional model evaluation tool. Data transformation was performed in R (V3.6.1(22)) and models were executed in conjunction with Perl-speaks-NONMEM (V4.8.1) (24).

\section{Study approval}

The study protocol was reviewed and approved by an independent medical ethics committee, the Medische Ethische Toetsingscommissie van de Stichting Beoordeling Ethiek Biomedisch Onderzoek (Assen, the Netherlands). All subjects provided informed consent prior to any study related procedures. The study was conducted at the Centre for Human Drug Research (Leiden, the Netherlands) from July 2018 until October 2018, and registered under clinical trial number NCT03607903. No interim analysis was performed.

\section{Results}

Forty-seven subjects underwent medical screening. Twenty-four subjects (male:female ratio 1:1) with Fitzpatrick skin type II were administered $40 \mathrm{mg}$ adalimumab (volume of $0.4 \mathrm{~mL}$ ) i.d. or s.c. in the lateral upper thigh and placebo (volume of $0.4 \mathrm{~mL}$ ) s.c. or i.d. in the contralateral thigh. One subject was randomized but excluded before treatment due to medical reasons and replaced (Figure 1 ). The mean age was 26.1 years (range 20-42). Demographic characteristics were comparable between groups (Table 1 ). For both s.c. and i.d. adalimumab injections a minor spill (1-15\% of intended volume not injected) occurred in 2 of $12(17 \%)$ injections, and there was one (8\%) major spill (15-50\% of intended volume not injected) in an i.d. adalimumab injection. Both the minor spills and the major spill during i.d. injection occurred when high resistance during injection was encountered, whereas the minor spill of s.c. injection was due to backflow. Inspection of the hollow microneedles after injection using bright field microscopy did not show damaged microneedle tips (not shown).

Pain, acceptability, and local tolerability after i.d. and s.c. adalimumab administration

Pain ascribed to needle injections is often divided into insertion pain which is pain resulting from the needle insertion, and injection pain which is pain resulting from the fluid injection. Insertion pain, injection pain, 
and post-injection pain were quantified using both a 100 points visual analogue scale (VAS) and the Dutch Faces Pain Scales Revised (FPSR, (15)). Insertion pain did not statistically significant differ between a hollow microneedle and a regular s.c. needle (Figure 2A , all $\mathrm{p}=0.22)$. Pain associated with fluid injection was higher for i.d. versus s.c. injections (Figure 2A, i.d. versus s.c. estimated means 29.5 and 8.3, decrease of $72 \%, 95 \%$ confidence interval $(\mathrm{CI})-83 \%-53 \%, \mathrm{p}<0.001)$. Intradermal adalimumab injections were more painful (estimated mean 35.4) than s.c. adalimumab injections (estimated mean 7.9). Comparing the treatments (placebo versus adalimumab, with both i.d. and s.c. administration methods combined) no statistically significant difference was observed $(\mathrm{p}=0.55)$. There was no difference within the administration method between adalimumab or placebo administration (placebo versus adalimumab within administration method $\mathrm{p}=0.32$ and $\mathrm{p}=0.81$ for i.d. and s.c., respectively). No pain was reported 24 hours after injection in any treatment group (Figure 2B ). For both insertion and injection pain a similar pattern in pain was reported in the FPSR in comparison with the VAS (data not shown). Altogether these subject reported outcomes indicate that there is no difference in pain between adalimumab and placebo injection, but that i.d. injection is more painful than s.c. injection.

To determine which injection type was preferred, subjects were asked about their preference: immediately after the injections (i.e., before seeing the injection area) and a day after the injections. Subject reported outcomes indicated that subjects had a preference for s.c. injection compared to i.d. injection (Figure 2C ). They also preferred to receive a hypothetical next injection using s.c. rather than i.d. administration (Figure 2D ). Directly after injection a majority (13 subjects, 54\%) indicated no fear, while 24 hours after injection most $(19,79 \%)$ subjects indicated no fear after injection. To summarize, we found that volunteers prefer s.c. over i.d. injection.

\section{Safety}

Nine treatment emergent adverse events were recorded; five in the s.c. group and four in the i.d. group. All treatment emergent adverse events were mild and self-limiting. Four subjects had fatigue, three had an upper respiratory tract infection, and one subject had a rhinitis. One subject had an injection site hematoma after i.d. adalimumab. Thus, i.d. and s.c. administration of adalimumab and saline do not raise a safety signal.

\section{Immunogenicity}

Anti-adalimumab antibodies are reported descriptively. None of the study participants had anti-adalimumab antibodies at baseline. Ten $(83 \%$, Figure 3A $)$ and six $(50 \%$, Figure 3B $)$ of the volunteers who received s.c. or i.d. adalimumab, respectively, treatment-emergent anti-adalimumab antibodies were detected. The median serum concentration for anti-adalimumab antibodies, for participants who developed anti-adalimumab antibodies, was 178 (range 16-864) for s.c. and 250 (range 189-940) arbitrary units for i.d. administration (Figure 3C ). Presence of anti-adalimumab antibodies was associated with increased adalimumab CL. However, high variability in the $\mathrm{AUC}_{0 \text {-inf }}$ was identified due to the differences in immunogenicity which needs to be taken into account to allow for a direct comparison of i.d. with s.c. administration.

PK of i.d. and s.c. adalimumab administration

The adalimumab concentration time profile is displayed in Figure 3D . First, a non-compartmental analysis of pharmacokinetics (PK) was performed. After exclusion of subjects where any leakage occurred during injection, in the remaining subjects $\mathrm{C}_{\max }$ was significantly higher after i.d. injection compared to s.c. injection (90\% CI 0.57-0.90, $\mathrm{p}=0.02)$. No difference was detected in $\mathrm{AUC}_{0 \text {-inf }}(90 \% \mathrm{CI} 0.55-1.09, \mathrm{p}=0.22)$ or $\mathrm{AUC}_{0 \text {-last }}$ (90\% CI 0.60-1.07, $\mathrm{p}=0.20$ ) (per protocol subjects in Table 2, all enrolled subjects inSupplementary table 1 ). These data show that i.d. administration of adalimumab yields a higher maximum concentration than s.c. administered adalimumab.

To further examine PK and to be able to correct for inter-individual variation in the kinetics of adalimumab and the formation of anti-adalimumab antibodies, a population PK model was developed. After exclusion of subjects in which any spill of adalimumab occurred during administration, data from 10 s.c. and 9 i.d. 
injections was available for model development using 275 adalimumab measurements that were above the lower limit of detection (LOD). A total of $4 \%$ of the measurements was below the LOD and therefore excluded from analysis. A significant effect between the time-varying titre levels and the CL was identified $(\mathrm{p}<0.001)$, indicating that the CL of adalimumab increases in the presence of high titre levels. However, a bias in the absorption kinetics for s.c. and i.d. was identified with linear absorption kinetics. Subsequent exploration of different structural absorption models resulted in a model event time (MTIME) function for the absorption rate constant $\left(\mathrm{k}_{\mathrm{a}}\right)$ after i.d. administration and two separate absorption compartments with equal $\mathrm{k}_{\mathrm{a}} \mathrm{s}$ and one with an absorption lag time for s.c. administration to be best fit for purpose (Figure 3E ). In this revised structural model, significant $(\mathrm{p}<0.01)$ inter-individual variability on the titre-CL relationship and the central volume of distribution was identified. Additionally, a significant $(\mathrm{p}<0.01)$ improvement in model fit was quantified after estimating a $29 \%$ higher bioavailability (F) after i.d. administration of adalimumab compared to s.c. administered adalimumab. A negative age-CL relationship and a positive weight-CL relationship were identified. Both covariates gave $\mathrm{p}<0.001$ improvement in the model fit. The developed model showed an overall accurate description of the absorption and elimination phase of adalimumab (Supplementary figure 2A-B ). Model parameters (Table 3 ) were estimated with high precision and were comparable to literature values (23). Simulations of the typical adalimumab absorption rates over time showed a clear difference between both administration routes, in which the i.d. dose had a fast initial phase which decreased after MTIME, whereas the s.c. administration had a slower initial phase and a small increase in the absorption rate, approximately 2 hours after dosing (Figure $\mathbf{3 F}$ ).

Cytokine production was assessed by stimulating ex vivo whole blood with LPS and aluminium hydroxide, driving NF $x$ B and NLRP3 inflammasome activation. Results are shown in Figure 4 . Free TNF $\alpha$ levels after both s.c. and i.d. administration sharply decreased from pre-dose to post-dose (mean levels pre-dose i.d. $897 \mathrm{pg} / \mathrm{mL}$, i.d. $48 \mathrm{~h}$ post-dose $50 \mathrm{pg} / \mathrm{mL}$, s.c. pre-dose $928 \mathrm{pg} / \mathrm{mL}$, s.c. $48 \mathrm{~h}$ post dose $74 \mathrm{pg} / \mathrm{mL}$ ), as has been reported earlier (16), and returned to baseline at the end of study (i.d. 70 days post-dose 1149 $\mathrm{pg} / \mathrm{mL}$, s.c. 70 days post dose $850 \mathrm{pg} / \mathrm{mL}$ ). No significant differences in inhibition of cytokine release were detected when i.d. adalimumab administration was compared to s.c. adalimumab administration (IFN $\gamma$ $\mathrm{p}=0.61 ; \mathrm{IL}-6 \mathrm{p}=0.31 ; \mathrm{IL}-8 \mathrm{p}=0.81 ; \mathrm{IL}-1 \beta \mathrm{p}=0.61 ; \mathrm{TNF} \alpha \mathrm{p}=0.80)$. For LPS/aluminium hydroxide induced IFN $\gamma$ production after adalimumab administration, a gender effect has been reported (14). A gender effect was not detected in this study (IFN $\gamma \mathrm{p}=0.99$, IL-6 p=0.80; IL-8 $\mathrm{p}=0.96$; IL-1 $\beta \mathrm{p}=0.75$; TNF $\alpha \mathrm{p}=0.08$ ).

Optical coherence tomography, clinical photography, thermal imaging, and laser speckle contrast imaging

Three-dimensional photography was used to quantify the bleb size after i.d. injection. No bleb formation was observed after s.c. injection. After i.d. injection bleb formation was observed after both adalimumab and saline injections, which resolved in less than a day (Figure 5A-B ). i.d. adalimumab administration but not s.c. adalimumab administration or injection of placebo caused local redness after injection (Figure 5C ). Optical coherence tomography was used to examine breach of epidermis and fluid disposition. Penetration of the epidermis was visible for $92 \%$ of cases 10 minutes after administration of both placebo injections and s.c. adalimumab injection. All i.d. adalimumab injections showed epidermal penetration 10 minutes post dose (Figure 5D-F ). Fluid disposition and vasodilatation in the dermis were visible more clearly for i.d. injections than s.c. injections.

Cutaneous microcirculation of the upper legs following injections was quantified using LSCI. A significant increase in blood flow for i.d. adalimumab injections compared to i.d. placebo, s.c. adalimumab, and s.c. placebo injections was shown 10 minutes post-dose $(\mathrm{p}<0.0001$, (Figure 5G )), followed by a decrease, reaching baseline on day 3 (data not shown). The bleb surface area was quantified using LSCI's perfused area measurements. The perfused areas were significant larger after i.d. adalimumab injections compared to i.d. placebo $(\mathrm{p}<0.0001)$, and also compared to s.c. adalimumab $(\mathrm{p}=0.0012)$ and placebo injections $(\mathrm{p}<$ 0.0001) (Figure 5H-I ).

Injection site temperature was measured in a temperature-controlled room using infrared thermography and corrected using standardized control areas (Supplementary figure 1 ). 


\section{In vitro protein analysis}

In vitro studies were performed to investigate whether passage of adalimumab through a hollow microneedle led to protein instability, as compared to passage through a regular s.c. needle. To this end, adalimumab was subjected to the same storage conditions and ejection methods as those used in the clinical trial. Protein conformational changes were determined by second-derivative UV spectroscopy, and formation of adalimumab aggregates and particles were determined by dynamic light scattering (DLS), high-pressure size exclusion chromatography (HP-SEC), Micro-Flow Imaging (MFI), and nanoparticle tracking analysis (NTA). Results of the protein analysis are shown inTable 4 . Second-derivative UV spectroscopy showed no change in $\mathrm{a} / \mathrm{b}$ ratio between conditions and time points, indicating no protein conformational changes. With DLS, no substantial differences in Z-average diameter were found. No substantial differences in the concentration of particles [?] $2 \mu \mathrm{m}$ were detected between conditions using MFI. NTA showed nanoparticle concentrations around the lower limit of detection $\left(10^{7}\right.$ ) (data not shown), and mean sizes were found ranging from 188 to $414 \mathrm{~nm}$. HP-SEC showed no differences in monomer content between conditions or between time points, and no evidence of aggregation or fragmentation. Molecular weights, based on multi angle laser light scattering (MALLS) data for the main peak, correspond to that of adalimumab reported before (20). These data show that passage of adalimumab through a hollow microneedle before storage and after storage for 4 hours at $2-8{ }^{\circ} \mathrm{C}$ does not lead to measurable protein aggregation or particle formation.

\section{Discussion}

With a sophisticated and comprehensive, multimodal PK-PD, safety approach we investigated a possibly minimally invasive administration method of adalimumab with a commercially available hollow microneedle. Importantly, this clinical trial shows that i.d. administration of a single dose of $40 \mathrm{mg}$ adalimumab in a volume of $0.4 \mathrm{~mL}$ using a hollow microneedle is safe and well accepted. However, i.d. administration was associated with an increased amount of injection pain and decreased volunteer preference compared to s.c. administration. Using imaging methods, the effect of i.d. injections on the skin was thoroughly characterized. As expected, i.d. injections led to bleb formation. Notably, i.d. injection transiently increased cutaneous microcirculation as measured by LSCI. Importantly, we found that i.d. administration of adalimumab led to a higher $\mathrm{C}_{\max }$ and a higher bioavailability compared to s.c. adalimumab administration. The inhibition of $e x$ vivo cytokine production of whole blood stimulated with LPS/Alum was similar for i.d. and s.c. adalimumab administration indicating comparable pharmacodynamic efficacy.

Protein degradation, especially aggregation, might result in increased immunogenicity of mAbs (11) and immunogenicity of mAbs is a major reason for secondary loss of response to mAbs. Therefore, we first showedin vitro that microneedle ejection of adalimumab does not substantially alters the amount of protein fragments or aggregates compared to ejection using a regular hypodermic needle.

Hollow microneedles are frequently considered a minimally invasive device to deliver parenteral drugs $(4,25-$ 28). In this study we administered a single adalimumab dose of $40 \mathrm{mg}$ in $0.4 \mathrm{~mL}$ or $0.4 \mathrm{~mL}$ placebo. We systematically studied pain associated with insertion and injection in a double-blind manner. We found that insertion pain of s.c. and i.d. administration was equal. However, injection pain of i.d. administration was significantly higher than s.c. administration. The high amount of pain is in contrast with another study, which used higher volumes but detected less pain (28). Pain due to s.c. injection is generally attributed to different factors, i.e., volume of injection, site of injection, formulation, needle size, and injection depth (29).

The volume limit of s.c. injection is generally considered to be $1.5 \mathrm{~mL}$ (30). Several studies have found higher volumes of s.c. administration to be associated with more pain (30-32). Thus, the increased pain that was associated with i.d. administration in the clinical trial reported in this paper is likely due to the volume injected. The volume used in this trial was limited by a minimum volume which contains a regular dose of a $\mathrm{mAb}$ in adults. Future studies might investigate the volume-pain relationship for i.d. administration using hollow microneedles. We did not detect a significant difference in pain when comparing adalimumab with placebo after i.d. and s.c. administration, which indicates that the formulation chosen in this study did not influence pain. 
Although not quantified, we observed a higher injection pressure during i.d. administration compared to s.c. administration. With OCT, we detected fluid filled cavities after i.d. injection, indicating there was no time for the compound to distribute in the skin.

We characterized the skin response to hollow microneedle administration of adalimumab using a combination of methods. The skin response following i.d. administration of adalimumab was mild and resolved within a day after injection. Using 3D photography, we showed the bleb which is typical for i.d. administration. Furthermore, using LSCI, an increase in cutaneous microcirculation after i.d. injection of adalimumab was observed. Our observations are of interest in the context of drug absorption. The increased cutaneous microcirculation might be associated with the increased adalimumab absorption following i.d. versus s.c. administration observed in our study. Yet, drugs injected s.c. may be absorbed via the lymph capillaries, or diffuse into blood capillaries, and after s.c. administration proteins with a high molecular weight, such as $\mathrm{mAbs}$, are predominantly absorbed via the lymph after s.c. administration $(33,34)$.

Various factors influence lymph flow, one being local skin temperature. During an increase in local skin temperature, both the blood flow and the lymph flow increase (35-37). We quantified local skin temperature after i.d. adalimumab administration using thermography. A limitation is that from the skin temperature measurements we cannot unequivocally conclude which type of injection (s.c. or i.d.) leads to higher skin temperature for two reasons. The temperature measurements might be confounded by difference in depth as i.d. injections are more superficial than s.c. injections. Thus, the s.c. injections might have increased the local temperature which is not apparent from our measurements.

Initial lymphatics, the part of the lymph vessels responsible for drug uptake, are located superficially, in the dermis (38). Under physiological conditions most of these lymph vessels are collapsed. Excess fluid (high hydrostatic pressure) and proteins (high local osmotic pressure) in the dermis cause high lymph flow. We used OCT to visualize epidermal penetration after i.d. injection. Qualitative analysis of OCT observations showed an increase in vessel diameter after i.d. injection compared to s.c. injection. Based on the OCT, no distinction can be made between blood and lymph vessels. Perhaps in the future a new variant of OCT, Doppler OCT (39), could be used to further characterize the physiology of mAb absorption and lymph flow.

Several studies have reported that the i.d. administration of drugs has different PK characteristics than s.c. delivery $(5,7,28,40)$. General observations are that $\mathrm{T}_{\max }$ is decreased, $\mathrm{C}_{\max }$ is increased and that bioavailability is either equal or increased after i.d. administration compared to s.c. administration. Most studies use insulin as model drug. For i.d. injection of insulin using hollow microneedles, it has been reported that $\mathrm{C}_{\max }$ increases and $\mathrm{T}_{\max }$ decreases after i.d. administration versus s.c. administration. It has been suggested that a shift in the concentration-time profile explains why some but not all studies have reported increased bioavailability after i.d. injection $(5,41)$. Changes in PK are generally attributed to anatomical differences in the skin: the dermis has extensive vasculature and lymphatics while the subcutis has more adipose tissue (42). When correcting for individual differences in the covariates and the titre values, this study showed a significant difference in bioavailability between s.c. and i.d. administration; i.d. administration was associated with a $29 \%$ higher bioavailability. In our study, a clear distinction in the absorption profiles over time could be observed between s.c. and i.d. administration. Adalimumab administered by microneedle injection show a short but fast absorption, whereas s.c. dosing shows a lower absorption rate. The steep drop in absorption after a microneedle injection is caused by the distribution of sampling points and an estimated mathematical time point. In reality, this transition would probably be smoother. Altogether, the PK profile of the i.d. administration of adalimumab is favourable over s.c. administration.

The immunogenicity of mAbs is a significant clinical problem hampering the treatment of autoimmune diseases with mAbs. In this study the number of healthy volunteers allows only for descriptive reporting of anti-adalimumab antibodies. The skin is a potent immune organ (42). Studies have shown an increased immunogenicity of i.d. vaccines compared to s.c. vaccines and microneedles are frequently studied as a device to deliver vaccines $(43,44)$. On the other hand, it has been suggested that i.d. administration of mAbs might lead to less immunogenicity compared to s.c. administration due to the presence of professional antigen presenting cells in the epidermis and dermis rather than in the subcutis $(33,45)$. Perhaps the relatively short 
residence time at the i.d. injection site of the (predominantly monomeric) protein might contribute to the lack of increased immunogenicity as compared to s.c. administration. It remains to be determined whether i.d. administration of biologicals alters the incidence, degree, or time of onset of anti-drug antibody formation compared to s.c. administration.

In this study the functional effect of adalimumab administration was investigated in vitro. Whole blood was stimulated with LPS/Alum and secreted cytokines were measured. We found that i.d. and s.c. adalimumab reduced ex vivo $\mathrm{TNF} \alpha$ bioavailability to a similar extent.

The increased bioavailability of i.d. adalimumab in our study suggests that lower doses may be used to achieve similar concentrations and subsequent effects compared to s.c. administration. Combined with the increased elasticity of the skin of children (46) and the need for a lower (adalimumab) dose than in adults, hollow microneedles ultimately might be suitable for use in paediatric patients. However, it is of paramount importance to better understand the pain-volume relationship of i.d. injections using hollow microneedles in adults first.

In conclusion, we showed that the i.d. administration of adalimumab is feasible and leads to faster absorption and increased bioavailability compared to s.c. administration. The amount of pain reported in this study, higher for i.d. than for s.c. adalimumab administration, is likely explained by the injection volume of 0.4 $\mathrm{mL}$. Understanding the relationship between pain and the administration of mAbs is essential before hollow microneedles can be investigated for use in the paediatric patient population.

\section{Acknowledgements}

We are indebted to our volunteers, and thankful to the team at the Centre for Human Drug Research and the pharmacy of the Leiden University Medical Center. Thanks to Karen Broekhuizen, medical writer at the Centre for Human Drug Research, who edited the manuscript for clarity.

Conflict of interest

Wim Jiskoot is a scientific advisor at Coriolis Pharma, Martinsried. Theo Rispens received fees for lectures from Pfizer, AbbVie, and Regeneron, and a research grant from Genmab. Koen van der Maaden is scientific advisor of MyLife Technologies B.V. and co-founder of uPRAX microsolutions B.V. The other authors have declared no conflict of interest exists.

\section{Funding information}

Funding: Dutch Arthritis Foundation: BP15-1-262.

\section{Data availability statement}

Research data are not shared.

\section{References}

1. Nelson AL, Dhimolea E, Reichert JM. Development trends for human monoclonal antibody therapeutics. Nat. Rev. Drug Discov. 2010;9(10):767-774.

2. Jacobse J, Ten Voorde W, Rissmann R, Burggraaf J, Ten Cate R, Schrier L. The effect of repeated methotrexate injections on the quality of life of children with rheumatic diseases. Eur. J. Pediatr.2019;178(1):17-20.

3. Donnelly RF, Raghu Raj Singh T, Larraneta. E, McCrudden MTC.Microneedles for Drug and Vaccine Delivery and Patient Monitoring. Chichester, UK: John Wiley \& Sons, Ltd; 2018.

4. Gupta J, Felner EI, Prausnitz MR. Rapid Pharmacokinetics of Intradermal Insulin Administered Using Microneedles in Type 1 Diabetes Subjects. Diabetes Technol. Ther. 2011;13(4):451-456. 
5. McVey E, Hirsch L, Sutter DE, Kapitza C, Dellweg S, Clair J, Rebrin K .. Pettis RJ. Pharmacokinetics and postprandial glycemic excursions following insulin lispro delivered by intradermal microneedle or subcutaneous infusion. J Diabetes Sci Technol. 2012;6(4):743-754.

6. Kochba E, Levin Y, Raz I, Cahn A. Improved Insulin Pharmacokinetics Using a Novel Microneedle Device for Intradermal Delivery in Patients with Type 2 Diabetes.Diabetes Technol. Ther. 2016;18(9):525-531.

7. Milewski M, Manser K, Nissley BP, Mitra A. Analysis of the absorption kinetics of macromolecules following intradermal and subcutaneous administration. Eur. J. Pharm. Biopharm. 2015;89:134-144.

8. Hróbjartsson A, Emanuelsson F, Thomsen ASS, Hilden J, Brorson S. Bias due to lack of patient blinding in clinical trials. A systematic review of trials randomizing patients to blind and nonblind sub-studies. Int. J. Epidemiol. 2014;43(4):1272-1283.

9. Levin Y, Kochba E, Kenney R. Clinical evaluation of a novel microneedle device for intradermal delivery of an influenza vaccine: Are all delivery methods the same? Vaccine2014;32(34):4249-4252.

10. Mahler SM, Marquis CP, Brown G, Roberts A, Hoogenboom HR. Cloning and expression of human $\mathrm{V}$ - genes derived from phage display libraries as fully assembled human anti-TNF $\alpha$ monoclonal antibodies.Immunotechnology 1997;3(1):31-43.

11. Hermeling S, Crommelin DJA, Schellekens H, Jiskoot W. Structure-immunogenicity relationships of therapeutic proteins.Pharm. Res. 2004;21(6):897-903.

12. Bartelds GM, Wijbrandts CA, Nurmohamed MT, Stapel S, Lems WF, Aarden L . . W Wolbink GJ. Clinical response to adalimumab: Relationship to anti-adalimumab antibodies and serum adalimumab concentrations in rheumatoid arthritis. Ann. Rheum. Dis. 2007;66(7):921-926.

13. Bartelds GM, Krieckaert CLM, Nurmohamed MT, Van Schouwenburg PA, Lems WF, Twisk JWR ... Wolbink JG. Development of Antidrug Antibodies Against Adalimumab and Association With Disease Activity and Treatment Failure During Long-term Follow-up. JAMA2011;305(14):1460.

14. Dillingh MR, Reijers JAA, Malone KE, Burggraaf J, Bahrt K, Yamashita L ... Moerland M. Clinical evaluation of humira $\mathbb{R}$ biosimilar ons-3010 in healthy volunteers: Focus on pharmacokinetics and pharmacodynamics. Front. Immunol. 2016;7:508.

15. Hicks CL, Von Baeyer CL, Spafford PA, Van Korlaar I, Goodenough B. The Faces Pain Scale - Revised: Toward a common metric in pediatric pain measurement. Pain. 2001;93(2):173-83.

16. Pouw MF, Krieckaert CL, Nurmohamed MT, Van Der Kleij D, Aarden L, Rispens T, et al. Key findings towards optimising adalimumab treatment: The concentration-effect curve. Ann Rheum Dis. 2015;74(3):5138.

17. Briers D, Duncan DD, Hirst E, Kirkpatrick SJ, Larsson M, Steenbergen W, Stromberg T, Thompson OB. Laser speckle contrast imaging: theoretical and practical limitations. J. Biomed. Opt. 2013;18(6):066018.

18. Everett JS, Budescu M, Sommers MS. Making Sense of Skin Color in Clinical Care. Clin Nurs Res. 2012;21(4):495-516.

19. Del Bino S, Bernerd F. Variations in skin colour and the biological consequences of ultraviolet radiation exposure. Br J Dermatol. 2013;169(SUPPL. 3):33-40.

20. Vlieland ND, Nejadnik MR, Gardarsdottir H, Romeijn S, Sediq AS, Bouvy ML ... Jiskoot W. The Impact of Inadequate Temperature Storage Conditions on Aggregate and Particle Formation in Drugs Containing Tumor Necrosis Factor-Alpha Inhibitors. Pharm. Res. 2018;35(42).

21. Kueltzo LA, Middaugh CR. Ultravioloet absorption spectroscopy. In: Jiskoot W, Crommelin DJA, editors. Methods for structural analysis of protein pharmaceuticals. Arlington: AAPS Press; 2005. 
22. R Core Team (2017). R: A language and environment for statistical computing. R Foundation for Statistical Computing, Vienna, Austria. URL: https://www.R-project.org/.

23. Berends SE, Strik AS, Van Selm JC, Löwenberg M, Ponsioen CY, D'Haens GR, et al. Explaining Interpatient Variability in Adalimumab Pharmacokinetics in Patients with Crohn's Disease. Ther Drug Monit. 2018;40(2):202-11.

24. Lindbom L, Ribbing J, Jonsson EN. Perl-speaks-NONMEM (PsN) - A Perl module for NONMEM related programming. Comput Methods Programs Biomed. 2004;75(2):85-94.

25. Kim YC, Park JH, Prausnitz MR. Microneedles for drug and vaccine delivery. Adv Drug Deliv Rev. 2012;64(14):1547-68.

26. Gill HS, Denson DD, Burris BA, Prausnitz MR. Effect of microneedle design on pain in human volunteers. Clin J Pain. 2008;24(7):585-94.

27. Kim YC, Park JH, Prausnitz MR. Microneedles for drug and vaccine delivery. Adv. Drug Deliv. Rev.2012;64(14):1547-1568.

28. Gupta J, Park SS, Bondy B, Felner EI, Prausnitz MR. Infusion pressure and pain during microneedle injection into skin of human subjects. Biomaterials. 2011;32(28)6823-31.

29. Usach I, Martinez R, Festini T, Peris JE. Subcutaneous Injection of Drugs: Literature Review of Factors Influencing Pain Sensation at the Injection Site. Adv Ther. 2019;36(11):2986-96.

30. Mathaes R, Koulov A, Joerg S, Mahler HC. Subcutaneous Injection Volume of BiopharmaceuticalsPushing the Boundaries. J. Pharm. Sci. 2016;105(8):2255-2259.

31. Heise T, Nosek L, Dellweg S, Zijlstra E, Praestmark KA, Kildegaard J ... Sparre T. Impact of injection speed and volume on perceived pain during subcutaneous injections into the abdomen and thigh: a singlecentre, randomized controlled trial. Diabetes, Obes. Metab.2014;16(10):971-976.

32. Jørgensen JT, Rømsing J, Rasmussen M, Møller-Sonnergaard J, Vang L, Musæus L. Pain assessment of subcutaneous injections. Ann Pharmacother. 1996;30(7-8):729-32.

33. Richter WF, Bhansali SG, Morris ME. Mechanistic determinants of biotherapeutics absorption following SC administration. AAPS J. 2012;14(3):559-70.

34. Supersaxo A, Hein WR, Steffen H. Effect of molecular weight on the lymphatic absorption of water-soluble compounds following subcutaneous administration. Pharm. Res.1990;7(2):167-9.

35. O'Morchoe CCC, Jones WR, Jarosz HM. Temperature dependence of protein transport across lymphatic endothelium in vitro. J Cell Biol. 1984;98(2):629-40.

36. Astrup A, Bülow J, Madsen J. Skin temperature and subcutaneous adipose blood flow in man. Scand J Clin Lab Invest. 1980;40(2):135-8.

37. Olszewski W, Engeset A, Icger PM, Sokolowski J, Theodorsen L. Flow and Composition of Leg Lymph in Normal Men during Venous Stasis, Muscular Activity and Local Hyperthermia. Acta Physiol Scand. 1977;99(2):149-55.

38. Ryan TJ, Mortimer PS, Jones RL. Lymphatics of the Skin: Neglected but Important. Int J Dermatol. 1986;25(7):411-9.

39. Blatter C, Meijer EFJ, Nam AS, Jones D, Bouma BE, Padera TP, Vakoc BJ. In vivo label-free measurement of lymph flow velocity and volumetric flow rates using Doppler optical coherence tomography. Sci. Rep.2016;6:29035.

40. Dul M, Stefanidou M, Porta P, Serve J, O'Mahony C, Malissen B, et al. Hydrodynamic gene delivery in human skin using a hollow microneedle device. J Control Release. 2017;265:120-31. 
41. Rini CJ, McVey E, Sutter D, Keith S, Kurth HJ, Nosek L, et al. Intradermal insulin infusion achieves faster insulin action than subcutaneous infusion for 3-day wear. Drug Deliv Transl Res. 2015;5(4):332-45.

42. Kabashima K, Honda T, Ginhoux F, Egawa G. The immunological anatomy of the skin. Nat. Rev. Immunol.2019;19(1):19-30.

43. Lambert PH, Laurent PE. Intradermal vaccine delivery: Will new delivery systems transform vaccine administration? Vaccine. 2008;26(26):3197-208.

44. Prausnitz MR, Mikszta JA, Cormier M, Andrianov AK. Microneedle-Based Vaccines. Curr Top Microbiol Immunol. 2009;333:369-93.

45. Büttel IC, Chamberlain P, Chowers Y, Ehmann F, Greinacher A, Jefferis R ... Schneider CK. Taking immunogenicity assessment of therapeutic proteins to the next level. Biologicals2011;39(2):100-109.

46. Rittié L, Fisher GJ. Natural and sun-induced aging of human skin.Cold Spring Harb. Perspect. Med. 2015;5(1):1-14.

Tables

Table 1: Demographics and baseline characteristics

\begin{tabular}{|c|c|c|}
\hline & All subjects $(\mathrm{N}=24)$ & All subjects $(\mathrm{N}=24)$ \\
\hline & i.d. $(\mathrm{N}=12)$ & s.c. $(\mathrm{N}=12)$ \\
\hline Age (years) & Age (years) & Age (years) \\
\hline Mean (SD) & $25.2(5.3)$ & $27.1(7.6)$ \\
\hline Median & 23 & 23.5 \\
\hline Min-max & $20-38$ & $20-42$ \\
\hline Height (cm) & Height (cm) & Height (cm) \\
\hline Mean (SD) & $177.8(6.1)$ & $180.1(7.6)$ \\
\hline Min-max & $167.3-188.5$ & 166.5-191.1 \\
\hline BMI $\left(\mathrm{kg} / \mathrm{m}^{2}\right)$ & BMI $\left(\mathrm{kg} / \mathrm{m}^{2}\right)$ & $\mathrm{BMI}\left(\mathrm{kg} / \mathrm{m}^{2}\right)$ \\
\hline Mean (SD) & $23.8(3.2)$ & $23.2(2.8)$ \\
\hline Min-max & $19.3-29.3$ & $20-28.8$ \\
\hline Sex & Sex & Sex \\
\hline Female (\%) & $6(25 \%)$ & $6(25 \%)$ \\
\hline Male $(\%)$ & $6(25 \%)$ & $6(25 \%)$ \\
\hline Race (\% per group) & Race (\% per group) & Race (\% per group) \\
\hline Asian & $0(0 \%)$ & $0(0 \%)$ \\
\hline Black or African American & $0(0 \%)$ & $0(0 \%)$ \\
\hline Mixed & $0(0 \%)$ & $0(0 \%)$ \\
\hline Other & $0(0 \%)$ & $0(0 \%)$ \\
\hline Caucasian & $12(100 \%)$ & $12(100 \%)$ \\
\hline
\end{tabular}

Table 2 Summary pharmacokinetic parameters for i.d. and s.c. adalimumab administration in the per protocol ${ }^{\mathrm{A}}$ study population.

\begin{tabular}{llllll}
\hline & s.c. $(\mathbf{N}=10)$ & s.c. $(\mathbf{N}=10)$ & s.c. $(\mathbf{N}=10)$ & i.d. $(\mathbf{N}=9)$ & i.d. $(\mathbf{N}=9)$ \\
\hline Parameter & Mean $(\mathrm{SD})$ & Median $(\min \max )$ & Mean $(\mathrm{SD})$ & Mean $(\mathrm{SD})$ & Median $(\min -\mathrm{max})$ \\
$\mathrm{C}_{\max }(\mu \mathrm{g} / \mathrm{mL})$ & $3.3(1.1)$ & $3.6(1.5-4.8)$ & $4.4(0.7)$ & $4.4(0.7)$ & $4.2(3.6-5.5)$ \\
$\mathrm{T}_{\max }(\mathrm{h})$ & $\mathrm{NA}$ & $120(96-221)$ & NA & NA & $95(47-120)$ \\
$\mathrm{AUC}_{0-\text { inf }}(\mu \mathrm{g} * \mathrm{~h} / \mathrm{mL})$ & $2359(1167)$ & $2048(853-5351)$ & $2986(1217)$ & $2986(1217)$ & $2724(1679-4897)$ \\
AUC $_{0-\text { last }}(\mu \mathrm{g} * \mathrm{~h} / \mathrm{mL})$ & $2189(816)$ & $2005(846-4019)$ & $2688(869)$ & $2688(869)$ & $2581(1677-4094)$ \\
\hline
\end{tabular}


$\mathrm{T}_{\max }$ : time to reach $\mathrm{C}_{\max } \cdot{ }^{\mathrm{A}}$ Subjects in the per protocol study population were subjects in which no leakage occurred and the intended dose of adalimumab was administered.

Table 3 Population pharmacokinetics parameter estimates with relative standard errors.

\begin{tabular}{|c|c|c|}
\hline Parameter & Estimate & RSE (\%) \\
\hline Absorption population parameters & Absorption population parameters & Absorption population parameter \\
\hline $\mathrm{F}_{\text {i.d. }}$ & 1.29 & 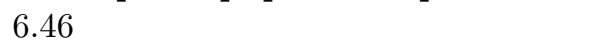 \\
\hline i.d. administration & i.d. administration & i.d. administration \\
\hline $\mathrm{k}_{\mathrm{a}-1}(/$ day $)$ & 3.54 & 10.1 \\
\hline $\mathrm{k}_{\mathrm{a}-2}(/$ day $)$ & 0.96 & 9.90 \\
\hline MTIME (days) & 0.078 & 9.32 \\
\hline s.c. administration & s.c. administration & s.c. administration \\
\hline $\mathrm{k}_{\mathrm{a}}(/$ day $)$ & 0.514 & 9.64 \\
\hline $\mathrm{F}_{\text {s.c. }-1}$ & 0.322 & 36.6 \\
\hline Lag time (days) & 0.075 & 36.7 \\
\hline Structural model parameters & Structural model parameters & Structural model parameters \\
\hline Volume of distribution central (L) & 11.5 & 8.02 \\
\hline CL (L/day) & 0.36 & 4.31 \\
\hline Covariate relationships & Covariate relationships & Covariate relationships \\
\hline CL-age exponent ${ }^{\mathrm{A}}$ & -0.70 & 24.3 \\
\hline CL-weight exponent ${ }^{\mathrm{B}}$ & 0.68 & 36.7 \\
\hline TITRE-slope (/xxx) & 0.064 & 25.1 \\
\hline Inter-individual variability & Inter-individual variability & Inter-individual variability \\
\hline$\omega^{2}$ Volume of distribution central & 0.069 & 31.2 \\
\hline$\omega^{2}$ TITRE-slope & 0.537 & 37.1 \\
\hline Residual variability & Residual variability & Residual variability \\
\hline$\sigma^{2}$ Proportional residual error & 0.054 & 13.9 \\
\hline
\end{tabular}

CL: clearance; F: bioavailability; i.d.: intradermal; $\mathrm{k}_{\mathrm{a}}$ : absorption rate constant; RSE: relative standard error; s.c.: subcutaneous.

A centered around 23 years. ${ }^{\mathrm{B}}$ centered around $70 \mathrm{~kg}$.

Table 4 Characterization of adalimumab after passage through a glass vial, a syringe, or a syringe with a hollow microneedle (Syr. $+\mathrm{MN})$, at 0 hours $(0 \mathrm{~h})$ and after storage at $4^{\circ} \mathrm{C}$ for 4 hours (4h). Representative data of two independent experiments.

\begin{tabular}{|c|c|c|c|c|c|c|c|}
\hline & $\begin{array}{l}\text { Time } \\
\text { point }\end{array}$ & $0 \mathrm{~h}$ & $0 \mathrm{~h}$ & $0 \mathrm{~h}$ & $4 \mathrm{~h}$ & $4 \mathrm{~h}$ & $4 \mathrm{~h}$ \\
\hline & Condition & Vial & Syr. & Syr. + MN & Vial & Syr. & Syr. + MN \\
\hline $\begin{array}{l}\text { UV } \\
\text { spectroscopy }\end{array}$ & $\mathrm{a} / \mathrm{b}$ ratio & 1.46 & 1.46 & 1.45 & 1.38 & 1.41 & 1.44 \\
\hline \multirow[t]{4}{*}{ DLS } & $\begin{array}{l}\text { Z-average } \\
\text { diameter }\end{array}$ & 3.76 & 3.68 & 3.99 & 3.59 & 3.61 & 4.23 \\
\hline & $\begin{array}{l}\text { in } \mathrm{nm} \\
(\mathrm{SD})\end{array}$ & $(0.01)$ & $(0.03)$ & $(0.33)$ & $(0.03)$ & $(0.07)$ & $(0.04)$ \\
\hline & $\begin{array}{l}\text { Polydispersity } \\
\text { index }\end{array}$ & 0.191 & 0.203 & 0.191 & 0.188 & 0.177 & 0.182 \\
\hline & $(\mathrm{SD})$ & $(0.003)$ & $(0.039)$ & $(0.002)$ & $(0.001)$ & $(0.080)$ & $(0.009)$ \\
\hline
\end{tabular}




\begin{tabular}{|c|c|c|c|c|c|c|c|}
\hline & $\begin{array}{l}\text { Time } \\
\text { point }\end{array}$ & $\mathrm{Oh}$ & $0 \mathrm{~h}$ & $0 \mathrm{~h}$ & $4 \mathrm{~h}$ & $4 \mathrm{~h}$ & $4 \mathrm{~h}$ \\
\hline \multirow[t]{3}{*}{ HP-SEC } & $\begin{array}{l}\text { Monomer } \\
\text { content (\%) }\end{array}$ & 99.8 & 98.0 & 98.0 & 99.6 & 99.6 & 99.6 \\
\hline & $\begin{array}{l}\text { Dimer } \\
\text { content }(\%)\end{array}$ & 0.2 & 2.0 & 2.0 & 0.4 & 0.4 & 0.4 \\
\hline & $\begin{array}{l}\text { Molecular } \\
\text { weight } \\
\text { monomer } \\
(\mathrm{kDa})\end{array}$ & 157 & 150 & 153 & 155 & 155 & 155 \\
\hline $\begin{array}{l}\text { NTA size } \\
\text { estimation }\end{array}$ & $\begin{array}{l}\text { Mean in nm } \\
\text { (SD) }\end{array}$ & $429(233)$ & $408(180)$ & $463(330)$ & $386(182)$ & $391(234)$ & $352(179)$ \\
\hline MFI & $\begin{array}{l}\text { Particles [?] } \\
2 \mu \mathrm{m} \text { per } \mathrm{mL}\end{array}$ & 3064 & 2948 & 2948 & 2434 & 2376 & 1773 \\
\hline
\end{tabular}

DLS: dynamic light scattering; HP-SEC: High-pressure size-exclusion chromatography; NTA: nanoparticle tracking analysis

MFI: Micro-Flow Imaging. UV spectroscopy, HP-SEC and MFI were measured with adalimumab samples diluted to $1 \mathrm{mg} / \mathrm{mL}$, DLS and NTA in a concentration of $10 \mathrm{mg} / \mathrm{mL}$.

\section{Figure legends}

Figure 1 CONSORT flow diagram of clinical trial. For PK and population PK analysis subjects in which any spillage occurred during injection were excluded. Other analyses were done with all subjects who completed the study $(\mathrm{N}=24)$.

Figure 2 Volunteer reported outcomes indicate preference for s.c. administration versus i.d. administration. Healthy volunteers were injected with a single dose of adalimumab in the upper thigh and placebo in the contralateral upper thigh administered i.d. using a hollow microneedle or s.c. using a conventional needle. Pain scores were measured during insertion of the needle (insertion pain) and during infusion of the compound (injection pain) using a 100 points VAS. Insertion and injection pain were normalized to the pain score during a Mantoux which the volunteers received during screening. (A) VAS pain scores for insertion pain. No differences were observed between s.c. and i.d. insertion pain ( $\mathrm{p}=0.68)$. (B) VAS pain scores for injection and post-injection pain. Injection pain was significantly $(p<0.0001)$ higher for i.d. compared to s.c. injection. Post-injection pain was not present. After injection, subjects were asked multiple choice questions about their preference, for (C) how they experienced the injection, (D) how they would like to get a hypothetical future injection, (E) and for which injection they had fear. (A-E): N=12 per group, except for Mantoux where $\mathrm{n}=24$. $(\mathrm{A}-\mathrm{B})$ : mean $\pm \mathrm{SD}$; repeated measures ANOVA; ${ }^{* * * *} \mathrm{p}<0.0001$. NA: not available because not measured. VAS: visual analogue scale.

Figure 3 Pharmacokinetics of adalimumab and anti-adalimumab antibodies after i.d. or s.c. Injection . Mean anti-adalimumab levels after (A) s.c. and (B) i.d. administration ( $\mathrm{n}=12$ per administration type). (C) Average anti-adalimumab levels for subjects with anti-adalimumab antibodies ( $\mathrm{n}=10$ for s.c. administration and $n=6$ for i.d. administration). (D) Serum adalimumab concentrations over time (D, $n=10$ for s.c. administration and $\mathrm{n}=9$ for i.d. administration, non-compartmental analysis). (C-D) Mean \pm SD. (E) Schematic depiction of population PK model. (F) Adalimumab absorption kinetics over time after adalimumab administration following microneedle (i.d.) or s.c. administration (typical population PK model). F: bioavailability; ID: intradermal; $\mathrm{k}_{\mathrm{a}}$ : absorption rate constant; SC: subcutaneous.

Figure 4 Similar cytokine production after i.d. or s.c. adalimumab administration (A) TNF $\alpha$, (B) IL-1 $\beta$, (C) IL-8, (D) IFN $\gamma$, and (E) IL-6 release after ex vivostimulation with LPS/aluminium hydroxide of 
whole blood samples. No gender effect was observed. Mean \pm SD. A-E: N=12 per group, repeated measures ANOVA.

Figure 5 Characterization of skin reaction following i.d. and s.c. injection. (A-C) 3D photography; (A) Typical bleb after i.d. injection. (B) Maximum height and volume of injection site. Volume was determined by outlining bleb circumference and height and calculated using the DermaPix (QuantifiCare, USA) algorithm for volume $(\sigma=5)$. Bleb height and volume did not differ between i.d. adalimumab and i.d. placebo (height $\mathrm{p}=0.26$, volume $\mathrm{p}=0.29$ ). (D) Redness of the injection sites, determined using a multispectral camera, displayed using the CIELAB *a ratio (green colours are negative, red colours positive). The more positive the CIELAB *a ratio, the redder the injection site. I.d. adalimumab and placebo injections induced significantly more redness of the skin compared to s.c. adalimumab and placebo injections $(\mathrm{p}<0.0001)$. Within i.d. administration, skin redness induced by adalimumab injection was significantly higher than for placebo injection $(\mathrm{p}=0.0014)(\mathrm{E}-\mathrm{F})$ Representative OCT images of i.d. injection 10 minutes post injection; (D-E) Cross-sectional planes of i.d. injection, and (F) top view of skin surface with three puncture holes. (G) LSCI was used to quantify skin perfusion in arbitrary PU 10 minutes post-injection. (H) Injection site surface area 10 minutes post injection. Area was calculated based on values above an arbitrary threshold of 90 PU. A significant difference in skin perfusion and surface area 10 minutes post injection was observed for both administration method $(\mathrm{p}<0.0001)$ and treatment $(\mathrm{p}<0.0001)$. (I) Representative LSCI images of both injection methods and treatments 10 minutes post injection. LSCI: laser speckle contrast imaging; OCT: optical coherence tomography; PU: perfusion units; B, D, G, H: mean $\pm \mathrm{SD}, \mathrm{N}=12$ per group, repeated measures ANOVA, ${ }^{* * * *} \mathrm{p}<0.0001$.

\section{CONSORT flow diagram}

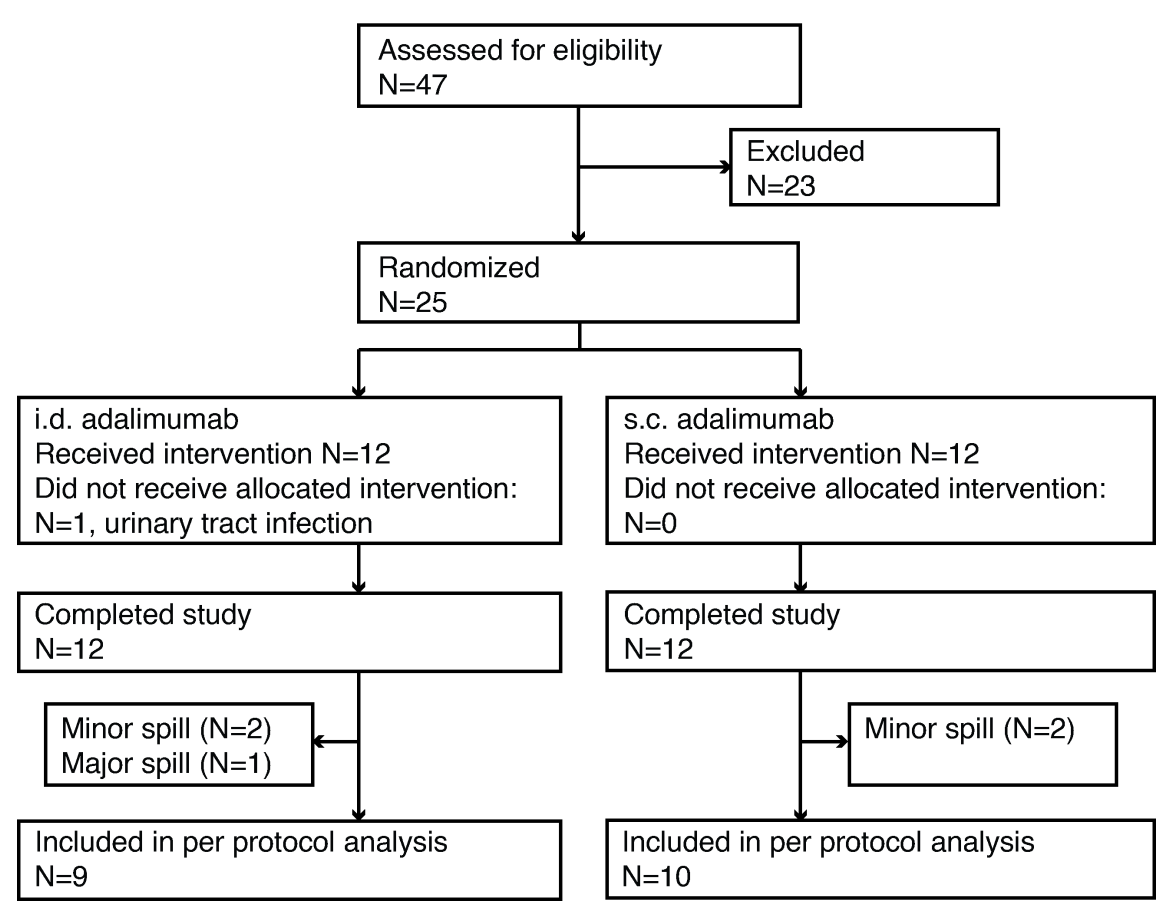




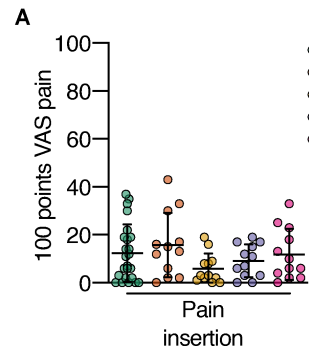

C

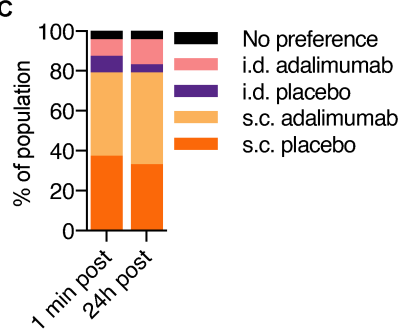

B

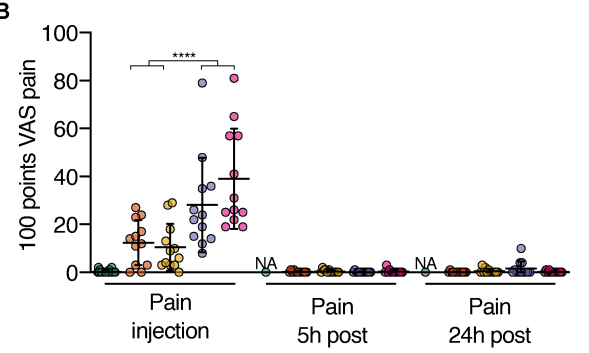

D

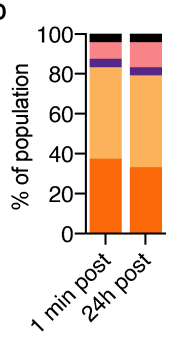

E

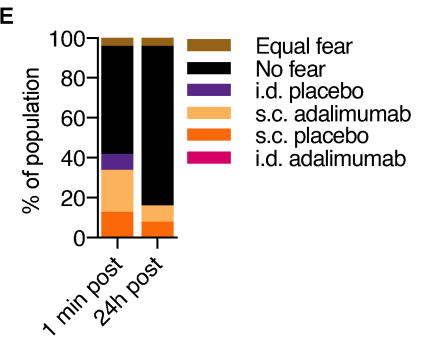

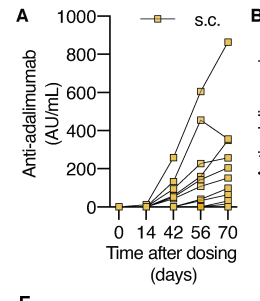

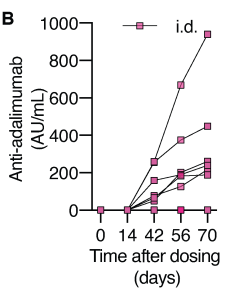

E

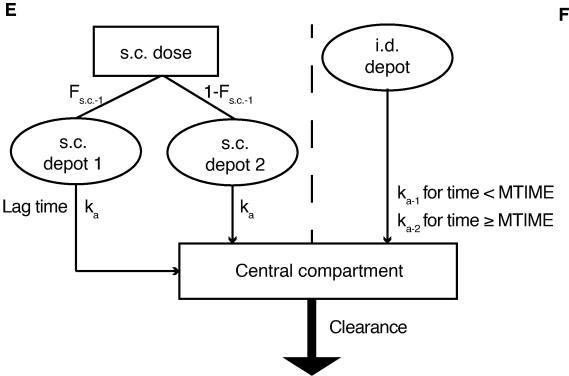

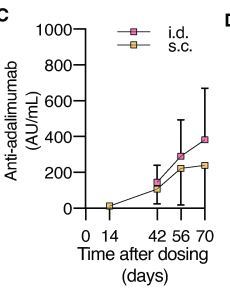
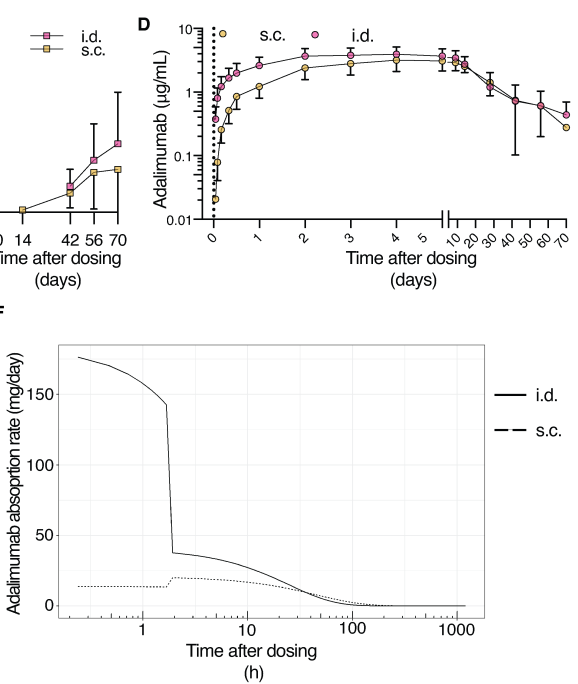

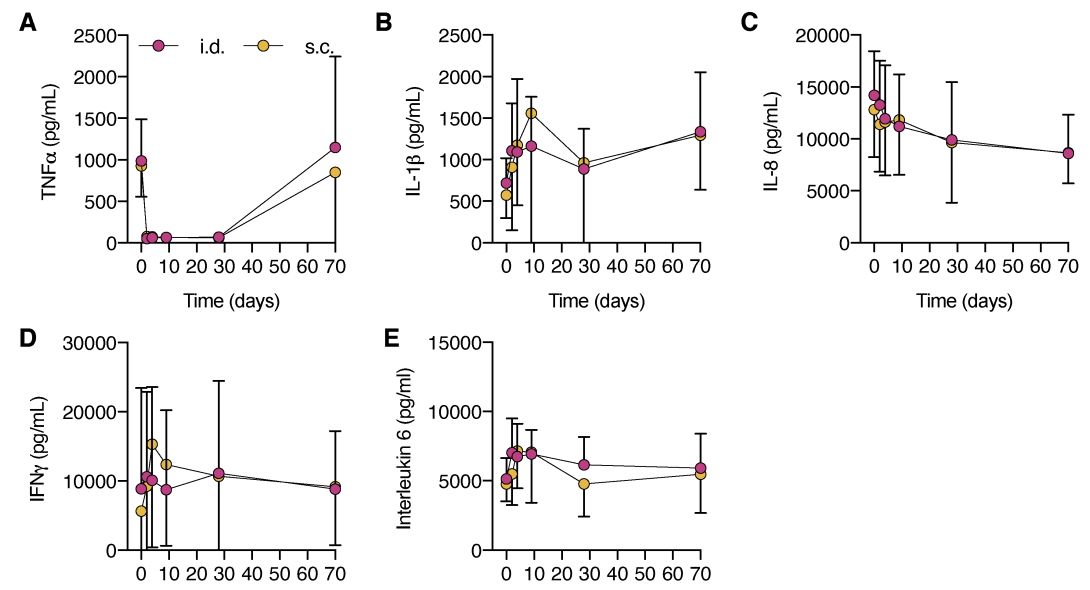

Time (days)
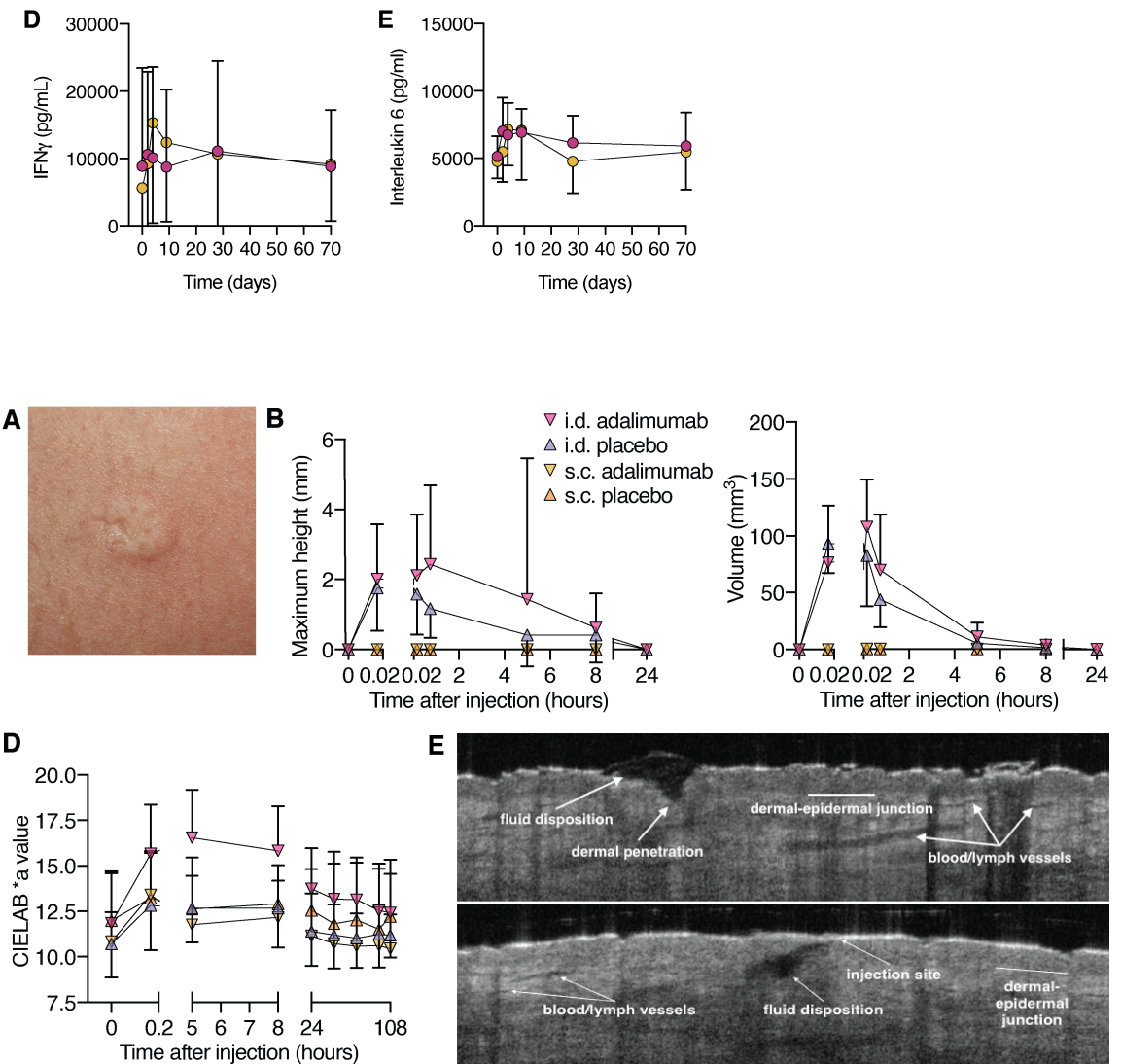

E

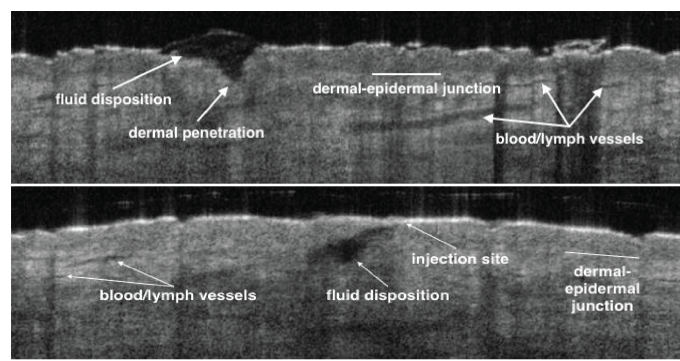

F

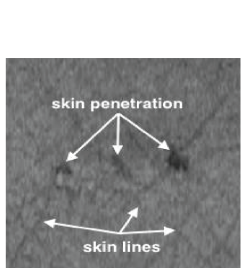

G
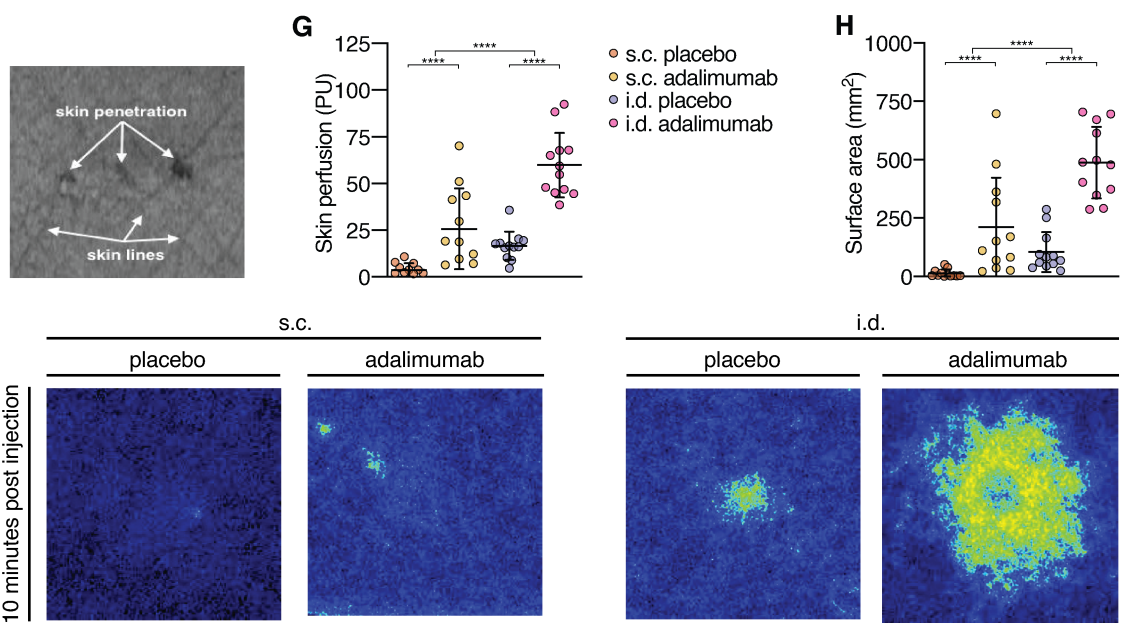

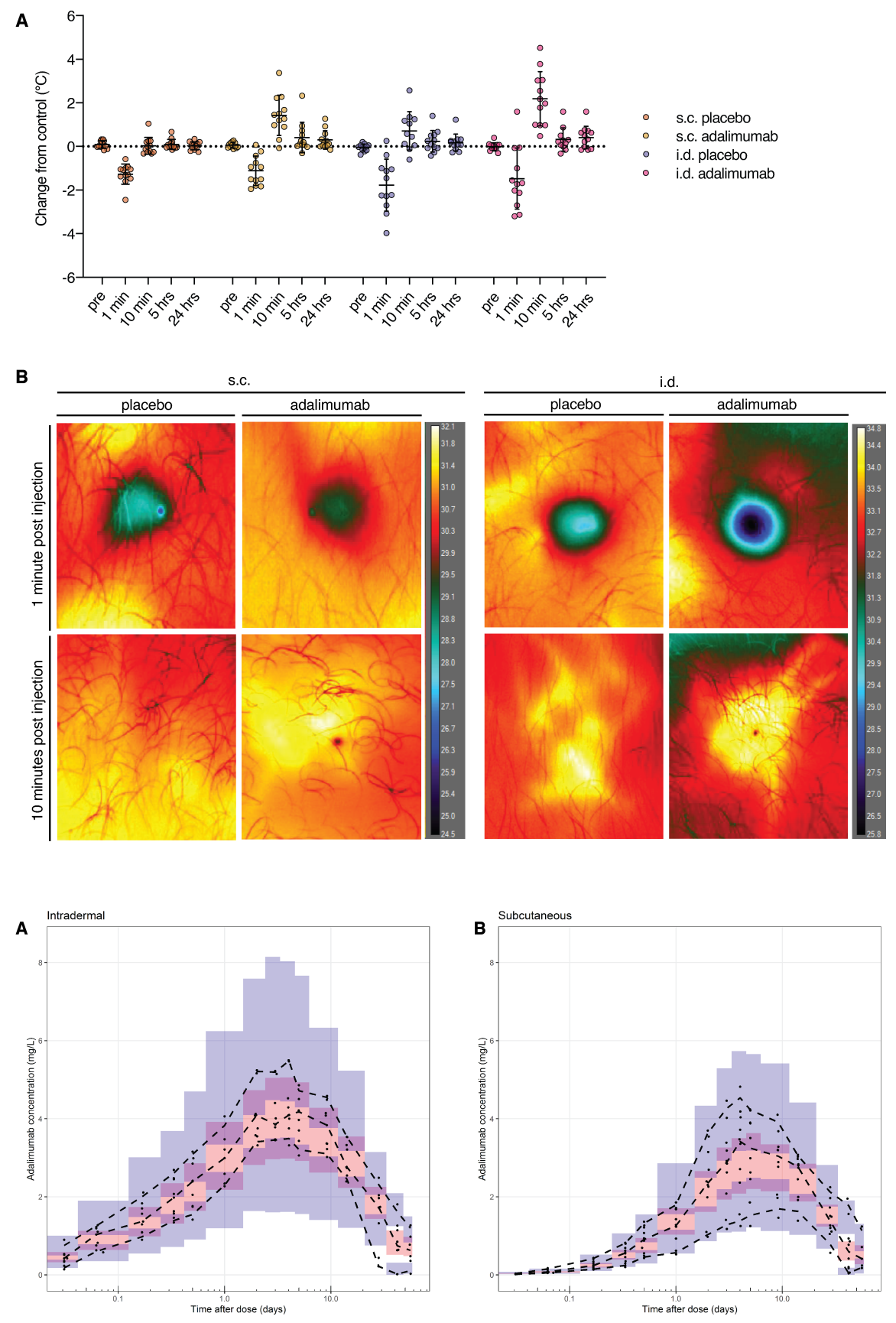